\title{
Erweiterung des Treuhandpartnernetzes in Winterthur
}

Die Dutler \& Partner Revision AG ist ein Treuhandunternehmen, das im Jahr 1997 gegründet wurde. Heute haben wir Standorte in Winterthur und Dielsdorf, wo insgesamt 7 Mitarbeiter arbeiten. Unsere Mitarbeiter zeichnen sich durch hohe fachliche Kompetenz in allen Treuhandbereichen aus. Mit gezielter Weiterbildung und ständigem Erfahrungsaustausch steigern wir die Fachkompetenz unserer Mitarbeiter, um für unsere Kunden den grösstmöglichen Nutzen zu erzielen.

Wir handeln nach ethisch einwandfreien Grundsätzen, ehrlich und innerhalb der gesetzlichen Schranken. Unsere Beziehungen zu unseren Kunden, Mitarbeitern, Partnern und Behörden sind geprägt durch Fairness, Verantwortungsbewusstsein und gegenseitige Wertschätzung als Basis für eine nachhaltige Zusammenarbeit.

Die Dutler \& Partner Revision AG ist Mitglied bei der Treuhand-Kammer und als zugelassene Revisionsexpertin bei der Revisionsaufsichtsbehörde registriert. Ebenfalls sind wir Partner bei der SRG Schweizerischen Revisionsgesellschaft AG und können so jeder-

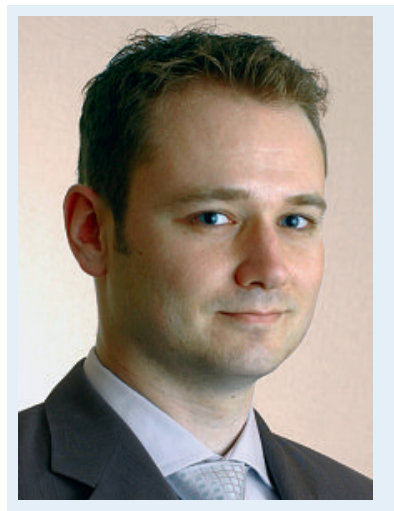

Andres Keller

Mitglied der Geschäftsleitung, Partner

Dipl. Treuhandexperte

Andres Keller hat über 10 Jahre Berufserfahrung im Treuhandbereich. Er ist ein ausgewiesener Spezialist für sämtliche Treuhandfragen. Er setzt sich kompetent und pflichtbewusst für die Anliegen der Kunden ein.

andres.keller@fmhtreuhand.ch zeit auf ein grosses Netzwerk an Spezialisten in allen Bereichen zurückgreifen.

Wir sind Ihr Partner bei

- sämtlichen Steuerangelegenheiten;

- der Einrichtung und Führung Ihrer Buchhaltung;

- der Personal- und Saläradministration;

- der Gründung Ihrer Unternehmung;

- der Unternehmensberatung;

- Erbschaftsangelegenheiten.

Andres Keller und Heinz Dutler sind Ihre FMH Treuhand Services-Ansprechpartner bei der Dutler \& Partner Revision AG. Sie freuen sich darauf, Ihr Unternehmen zusammen mit Ihnen in eine erfolgreiche Zukunft zu führen.

\section{Dutler \& Partner Revision AG FMH Treuhand Services \\ Theaterstrasse 17 \\ $\mathrm{CH}-8400$ Winterthur}

Tel. 05220908 12, Fax 0522090819

www.dup.ch

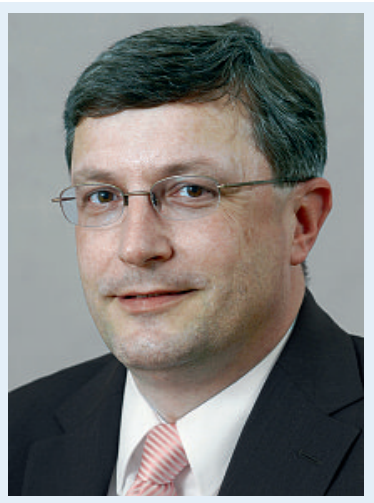

\section{Heinz Dutler}

Präsident des Verwaltungsrates, Partner

Dipl. Treuhandexperte

Heinz Dutler hat über 30 Jahre Berufserfahrung im Treuhandbereich. Er ist ein ausgewiesener Spezialist für sämtliche Treuhandfragen. Das Unternehmertum liegt ihm im Blut, und für pragmatische Lösungsansätze ist er bekannt.

heinz.dutler@fmhtreuhand.ch 